\title{
Non-Hodgkin Lymphoma with Variable Clinical Course
}

National Cancer Institute

\section{Source}

National Cancer Institute. Non-Hodgkin Lymphoma with Variable Clinical Course. NCI

Thesaurus. Code C7216.

A non-Hodg kin lymphoma with variable clinical course. 